\title{
Down Syndrome Cell Adhesion Molecule, Mother of Innovations
}

\author{
Brian E. Chen
}

doi:10.1017/cjn.2015.235

Can J Neurol Sci. 2016; 43: 52-55

The pleasure of starting up a laboratory in Canada is one of the most famous secrets in science. The exhilarations are akin to a rebirth at the stage in life for a new investigator to reflect on achievements, the present enjoyment, and the anxious anticipation of embarking on a new path. Research in Canada comes with no pretense and allows an investigator to bring to it what you will. The Canadian research atmosphere is calm and risk-aversive, an appropriate representation of Canada. This allows researchers to do their best research by putting into place any resources that they may need without extravagance, but without flair. For example, all independent investigator positions across Canada are "hard money" positions, meaning their salaries are guaranteed by their host institution and not apportioned from or dependent on receiving grant funding. But even with assured personal financial security, there are few award mechanisms dedicated to high-risk, high-reward research, nor "people not projects" funds to foster flexibility and creativity.

New investigators start their laboratories by learning the particulars of their country's research funding systems. In Canada, most funding for neuroscience research comes from two federal agencies, the Canadian Institutes of Health Research and the Natural Sciences and Engineering Research Council of Canada. Because the burden of principal investigator (PI) salaries are not entirely captured by these agencies' budgets, it is not entirely accurate to compare budgets, but comparisons to the United States are simplified because Canada is roughly one-tenth in population (35 million vs 316 million people, or 11\%) and gross domestic product (GDP, CAN\$1.8 trillion vs US\$16.8 trillion, or $11 \%){ }^{1}$ The annual budget of the Canadian Institutes of Health Research is US\$1 billion, or $0.06 \%$ of the Canadian GDP, and the annual budget of the US National Institutes of Health is US\$30 billion, or $0.18 \%$ of the US GDP. ${ }^{2,3}$ Funding rates for Canadian grants tend to be much higher than in the United States and tend towards a more egalitarian sharing funding system across Canada. ${ }^{4}$

A common challenge to new investigators is attracting talented personnel to a new laboratory. It can be a long time before potential personnel aim directly for joining an investigator's laboratory, rather than discovering the new investigator through the host institution and its reputation first. New Canadian investigators compete with established laboratories as well as the lure of United States laboratories that attract talented young scientists. Fortunately, being the next generation of PIs should have its advantages, and new PIs were presumably hired to expand new and exciting science and be on the cutting edge of their fields. With the benefits of academic freedom, depending on an investigator's institution, these new creative ideas can be pursued, and new PIs can leverage the internet and social media to advertise these attractions while adding their personality and vision to their research goals - their brand, if you will. The appeal of living and working in Canada can also be highlighted through these personalized, directed outlets, and the strength of Canadian neuroscience can be showcased through professional societies, such as the Canadian Association for Neuroscience.

An exciting challenge that starting a new laboratory brings is choosing long-term interesting and impactful questions while balancing the pressure of short-term productivity - the reassuring feeling of validation. Limited funds invariably temper this urgency for growth and keep new laboratories lean, hungry start-up environments. Fortunately, in discovery-based research, there will always be new and interesting questions to answer; the threat of competition is simply a sign of lack of creativity. The freedom is exciting, and paving a new path provides opportunities to define yourself and crystalize your laboratory and vision while knowing you will be guiding the path of many to come through mentorship, employment, and knowledge creation. Academic freedom lets you explore and pursue your creative visions and allow you to flourish. The exhilarations come from knowing that the only restrictions you have are external funding and your internal creativity and leadership abilities. Neuroscience is the undiscovered country. Being a neuroscience researcher is to investigate the most complex machine in the known universe. What is it like to be a new investigator? It is thrilling.

\section{An Interesting Problem: Neural Wiring}

The question I decided to pursue is how hard-wired neural circuits can be created using genetic instructions. How does nature build a brain that can completely self-assemble and function as soon as an animal is born? Over the past few decades, we have learned much about how neural circuits refine through activity-dependent plasticity, such as for learning and memory. However, we know little about how innate behaviors, such as breathing or reflexes, are prespecified in hard-wired circuits. Even a "simple" neural circuit with hundreds of neurons can contain millions of synaptic connections between them. This synaptic connectivity must also be

From the Centre for Research in Neuroscience, Research Institute of the McGill University Health Centre, Montréal, Québec, Canada, Departments of Medicine and Neurology \& Neurosurgery, McGill University, Montréal, Québec, Canada

Received March 3, 2015. Final Revisions Submitted May 1, 2015.

Correspondence to: Brian Chen, Centre for Research in Neuroscience, Research Institute of the McGill University Health Centre, Montreal General Hospital, 1650 Cedar Ave, L7-224, Montréal, Québec, H3G 1A4, Canada. Email: brian.chen@ mcgill.ca 
extremely precise for any hard-wired circuits that underlie behaviors that affect the fitness of the animal, such as an escape response. The assembly instructions for hard-wired circuits must be written molecularly within the genome, and my research focuses on deciphering these instructions to uncover the molecular codes and strategies that neurons use to connect with each other. I chose to investigate this question to help us understand the basic rules governing how genetic information translates into the synaptic targeting decisions of neurons to produce innate behaviors.

\section{DSCAM, Mother Nature's InNOVATion}

My introduction into how molecular information can specify the synaptic targeting decisions of a neuron came from investigating a gene called Down Syndrome Cell Adhesion Molecule (Dscam) in the fruit fly genetic model organism Drosophila melanogaster. DSCAM is was discovered on human chromosome band $21 \mathrm{q} 22$, within a 4-Mb region called the Down syndrome critical region. ${ }^{5}$ Because the human gene was predicted to encode a large receptor with multiple immunoglobulin domains, it was named the Down Syndrome Cell Adhesion Molecule. The Drosophila Dscam protein is identical in domain structure to human DSCAM, placing Dscam's origin at more than 600 million years ago. However, Drosophila Dscam is a very complex molecule with a unique feature: through alternative splicing, the gene can produce more than 100,000 different protein isoformsthe record holder for alternative splicing. ${ }^{6,7}$ Different RNA isoforms are created by splicing together different alternate exons within the gene, and each of these RNA isoforms is translated into protein isoforms that all maintain the same overall Dscam protein architecture. This extensive isoform diversity has been proposed as a synapse-specific molecular label through different binding affinities of Dscam isoforms. ${ }^{8}$ To determine whether Dscam plays a role in synapse specification, I used a stereotyped axonal branching pattern as a readout for errors in synaptic targeting. By combining this with single-neuron genetic mosaic analysis and exon-specific Dscam deletions, I showed that Dscam's extensive isoform diversity is required for proper synaptic targeting at the level of single axonal branches and that different isoforms are used to specify different synaptic contacts. ${ }^{9}$ This study from my postdoctoral research opened the possibility that complex neural circuits can be prespecified genetically using diverse molecular labels generated by alternative splicing rather than being refined through the experience of the animal. This work was the foundation for the research in my laboratory investigating how hardwired neural circuits wire up.

Dscam has now been shown to be an important molecule involved in many aspects of neural development in invertebrates and vertebrates including axon guidance, axonal and dendritic branching, synapse targeting, synaptic plasticity, and cell death (reviewed elsewhere ${ }^{7,10}$ ). However, vertebrate DSCAM is not extensively alternatively spliced, and my long-term goal is to understand how hard-wired neural circuits are molecularly prespecified, particularly in the mammalian brain. I use the Drosophila fruit fly as a tractable model system and investigate Drosophila Dscam hoping to reveal general strategies about how neural circuits use a diversity of cellsurface receptors to specify precise synaptic contacts. These insights also help guide my laboratory's investigations into vertebrate mechanisms of neural wiring. Other laboratories have discovered parallel mechanisms - for example, in neurite self-avoidance,
Drosophila Dscam isoform diversity or mammalian Protocadherin isoform diversity is used by neuronal branches to avoid self-crossing (reviewed elsewhere ${ }^{11,12}$ ). For me, this has left more questions than answers about the need for isoform diversity because not all complex neurons and neural circuits require it. Why does the Pancrustacea clade (comprising crustaceans and hexapods, the majority of the animal biomass on the planet) all require such extraordinarily large isoform diversity of Dscam? $?^{7,13,14}$ What, then, are the genetic instructions to prespecify a hard-wired complex neural circuit in mammals? In the end, why investigate Drosophila Dscam if there are no human genes (mammalian protocadherins generate around 50 isoforms) with remotely similar extensive alternative splicing?

Dscam is the most complicated gene ever discovered. Here I provide brief examples of how curiosity-driven research on Dscam has pushed innovation in my laboratory and produced unexpected and far-reaching outcomes, often beyond neuroscience. Much of these advances arose simply because of the size and complexity of the Dscam gene. The Drosophila Dscam gene is $75 \mathrm{~kb}$ with 25 exons, but within variable exons $4,6,9$, and 17 , there exist tandem arrays of nearly 100 alternate exons. ${ }^{7}$ Thus, performing or proposing experiments on Dscam forces you to think big. No meaningful experiments can be performed on the standard scale because of the hundreds of thousands of isoforms confounding the interpretation. Standard protein structure-function analyses are useless because of the absurd number of protein isoforms to manipulate. Loss of function or necessary and sufficiency analyses are equally difficult because of the broad range of phenotypes that may result from compensating or misregulated isoforms. Thus, it is nearly impossible to ascribe any specific function to specific isoforms. These theoretical and conceptual complexities become real-world challenges when there are limited funds for newly starting, unproven investigators studying fruit flies. Here it becomes even more critical to understand what impactful and interpretable result will come and what significant question will be answered at what monetary and time cost. But the riddle of Dscam will always be a fascinating problem to solve.

\section{Down Syndrome CELL AdHesion Molecule AND NeURAL Wiring: Implications for Down Syndrome ANd Fragile X SYNDROME}

Dscam has been shown to be involved in several phenotypes associated with Down syndrome. ${ }^{15-17}$ Down syndrome is the result of triplication for human chromosome 21, which causes an overexpression of the thousands of genes located there. Similarly, Fragile $\mathrm{X}$ syndrome is the result of an overexpression of thousands of genes resulting from the silencing of the Fragile $X$ Mental Retardation gene. This gene encodes Fragile X Mental Retardation Protein (FMRP), a suppressor of protein translation; excessive protein synthesis of FMRP's thousands of normally suppressed messenger RNA (mRNA) targets is thought to underlie the neuronal defects in Fragile X syndrome. Interestingly, DSCAM mRNA was found to be selectively associated with FMRP (mouse and human) in high-throughput RNA sequencing and microarray screens identifying molecules involved in Fragile $X$ syndrome and autism spectrum disorders. ${ }^{18-20}$ Thus, my laboratory sought to determine whether overexpression of Drosophila Dscam protein through gene triplication (reflecting the Down syndrome trisomy 21 case) or through loss of translational suppression by FMRP impairs synaptic targeting precision and neural circuit function. ${ }^{21}$ 
To examine how excessive Dscam protein levels affect neural wiring decisions, we used a hard-wired mechanosensory circuit in Drosophila that allows for quantitative analysis of synaptic targeting errors. ${ }^{9,22,23}$ This mechanosensory system also allows for a functional assessment of synaptic connectivity through the animal's reflex behavior by mechanically stimulating the neuron. ${ }^{23}$ Using Drosophila genetics, we removed FMRP solely within four mechanosensory neurons, leaving the rest of the animal and nervous system normal. We found that this resulted in reproducible synaptic targeting errors within just these mechanosensory neurons and disrupted the animal's mechanosensory perception. Animals with three copies of the Dscam gene had similar synaptic targeting errors and impaired touch responses as those with loss of FMRP. Reducing the Dscam gene dosage in the FMRP mutant animals reduced the synaptic targeting errors and rescued the behavioral responses, indicating that much of the errors in neural wiring and synaptic function were the result of excess Dscam protein in these neurons. We were also curious as to whether FMRP regulated all Dscam RNA isoforms. We used next-generation sequencing to obtain long read lengths of nucleic acids and deep coverage of more than 1 million reads and found that all possible isoforms were equally associated with FMRP, with no significant bias in the Dscam isoforms bound. This study demonstrated that excess Dscam protein might be a common molecular mechanism underlying altered neural wiring in two of the most common causes of intellectual disability: Down syndrome and Fragile $\mathrm{X}$ syndrome. $^{21}$ Because Dscam function is evolutionarily conserved across vertebrates and invertebrates in both cardiac and neural development, ${ }^{7,24}$ we speculate that Dscam protein expression is tightly regulated in cells, and dysregulation in neurons may underlie a wide variety of neural developmental disorders.

\section{How Does Protein Concentration Affect Neural Phenotypes?}

To examine how dysregulation of mammalian DSCAM protein might affect cortical development, we sought to overexpress DSCAM in mouse neocortical neurons. It would be beneficial to understand how excess DSCAM protein concentration produces different abnormal neural phenotypes and, ideally, how the variations in concentration of endogenous DSCAM protein might correlate with the variations in these same phenotypes in normal neurons. Although DSCAM is expressed at high levels in the developing neocortex, ${ }^{25}$ the natural heterogeneity of cortical neurons and their large variability in phenotypes-for example, in dendritic branching structure and ion channel properties ${ }^{26,27}-$ precludes any meaningful analysis of DSCAM expression with neural phenotype at the single-neuron level, unless the effect size is large or the neurons are as stereotyped as Drosophila neurons.

Thus, we needed a method to correlate the variation in neural phenotypes with concentration of DSCAM protein at the singlecell level. Accurate quantification of protein amounts is very difficult at this level. The only current ways to quantify protein levels are using crude techniques such as protein assays and immunoblots. Essentially, these entail dissecting and homogenizing heterogeneous tissue from large numbers of animals, sometimes from irrelevant developmental stages or tissues because of widely accepted dissection practicalities. Because of poor sensitivity and detection capabilities, these techniques become laborious and time-consuming, but also less relevant because of their poor cellular resolution. All current techniques are also necessarily destructive to cells and animals. To overcome these limitations, we developed a technique to quantitate protein amounts in single living cells, which we call "protein quantitation ratioing." This technique uses modified virus sequences that allow for an equimolar separation of an upstream protein of interest and a downstream protein of interest, all contained within a single strand of RNA. When a fluorescent reporter is separated from the protein of interest, the number of fluorescent molecules produced is stoichiometric with the number of molecules of the protein of interest produced, and thus the fluorescence output (i.e. brightness of the cell) can be used as a readout for the number of molecules of interest produced (i.e. its relative protein concentration). ${ }^{28}$ We invented this technique to correlate DSCAM protein concentration with neural phenotype in single neurons in the living animal. By simply using the cell's brightness as a readout for the protein expression level of a gene, we hope that our protein quantitation ratioing technique will have a wide range of creative applications in cell biology to quantitatively measure relationships between phenotypes and protein levels, with broad expansion possibilities. Almost every cell biologist requires the quantification of protein amounts at some point; thus, our technique could have a large impact across biology.

\section{Visualizing and Browsing the Most Complicated Gene EVER Discovered}

Performing experiments on Drosophila Dscam is difficult because of the complexity of the alternative splicing. Even visualizing the gene itself is a challenge, whether when designing experiments or explaining the gene's alternative splicing to laboratory trainees. To ease this burden, we created a web application for online visualization and browsing of the different alternatively spliced RNA and protein isoforms (brianchenlab. mcgill.ca/dscam). This web app allows for visual selection of isoforms by moving the cursor over the desired alternate exon, which will then dynamically update and display the corresponding mRNA and amino acid sequences. An interactive protein schematic of Dscam is also displayed above the mRNA and amino acid sequences for structure-function experiments, so that specific protein domains (e.g. immunoglobulin domain 7) can be selected and the corresponding mRNA and amino acid sequences are highlighted. These are only a few of the major features that are included in our web app, designed and deployed within one summer. Seeing this as inspiration, I realized that if this could be accomplished for the most complex Drosophila gene, then it could be accomplished for the entire Drosophila genome and, by extension, all sequenced genomes available; soon afterwards GeneDig.org was created. Both of these web tools were developed because of the striking lack of resources available for accessing genomic and bioinformatics data for standard biology applications.

GeneDig is a web application for user-friendly access to genomics and bioinformatics knowledge, ${ }^{29}$ and is designed to be comprehensive yet intuitive, allowing for searching and browsing of all sequenced genes and genomes with ease and efficiency. Our dynamic navigator displays genomic, RNA, and protein information simultaneously for co-navigation, with disease and traits overlaid on associated genes. GeneDig is freely available in more than 15 languages at GeneDig.org. I created GeneDig to benefit 
any general user that requires information about specific genes or diseases, for scientists, health care practitioners, patients, or science education. I believe that access to genomic information is important, and my goal with GeneDig is to make all genomic information easily accessible and useful.

\section{Conclusion}

There has never been a better time to be a new investigator in neuroscience. Rapid advances in research and technologies as well as a strong neuroscience community in Canada and worldwide push the field to the forefront of human endeavors. The mysteries of the brain will continually produce fun and interesting challenges for scientists, and unconventional research forces you to think differently. My roadmap for the formative years of my laboratory was the DSCAM gene, but only as it is an intrinsic curiosity. I wholeheartedly recommend that approach: solve interesting problems. Difficult problems will birth creative solutions.

\section{Disclosures}

$\mathrm{BC}$ is a researcher for and has received research support from the Canadian Institutes of Health Research and Natural Sciences and Engineering Research Council of Canada.

\section{REFERENCES}

1. GDP. World Development Indicators. Available from: http://data. worldbank.org/indicator/NY.GDP.MKTP.CD: The World Bank; 2013.

2. A Portfolio for Health Innovation-Canadian Institutes of Health Research Annual Report 2013-14. Available from: http://www. cihr-irsc.gc.ca/e/48774.html.

3. NIH Budget. Available from: http://www.nih.gov/about/budget.htm.

4. Ioannidis JP. More time for research: fund people not projects. Nature. 2011;477:529-31.

5. Yamakawa K, Huot YK, Haendelt MA, et al. DSCAM: a novel member of the immunoglobulin superfamily maps in a Down syndrome region and is involved in the development of the nervous system. Hum Mol Genet. 1998;7:227-37.

6. Schmucker D, Clemens JC, Shu H, et al. Drosophila Dscam is an axon guidance receptor exhibiting extraordinary molecular diversity. Cell. 2000;101:671-84.

7. Schmucker D, Chen BE. Dscam and DSCAM: complex genes in simple animals, complex animals yet simple genes. Genes Dev. 2009;23:147-56.

8. Schmucker D, Flanagan JG. Generation of recognition diversity in the nervous system. Neuron. 2004;44:219-22.

9. Chen BE, Kondo M, Garnier A, et al. The molecular diversity of dscam is functionally required for neuronal wiring specificity in drosophila. Cell. 2006;125:607-20.

10. Montesinos ML. Roles for DSCAM and DSCAML1 in central nervous system development and disease. Adv Neurobiol. 2014; $8: 249-270$.
11. Kise Y, Schmucker D. Role of self-avoidance in neuronal wiring. Curr Opin Neurobiol. 2013;23:983-9.

12. Zipursky SL, Grueber WB. The molecular basis of self-avoidance. Annu Rev Neurosci. 2013;36:547-68.

13. Brites D, Brena C, Ebert D, Du Pasquier L. More than one way to produce protein diversity: duplication and limited alternative splicing of an adhesion molecule gene in basal arthropods. Evolution. 2013;67:2999-3011.

14. Chipman AD, Ferrier DE, Brena $\mathrm{C}$, et al. The first myriapod genome sequence reveals conservative arthropod gene content and genome organisation in the centipede Strigamia maritima. PLoS Biol. 2014;12:e1002005.

15. Alves-Sampaio A, Troca-Marin JA, Montesinos ML. NMDAmediated regulation of DSCAM dendritic local translation is lost in a mouse model of Down's syndrome. J Neurosci. 2010;30: $13537-13548$

16. Barlow GM, Chen XN, Shi ZY, et al. Down syndrome congenital heart disease: a narrowed region and a candidate gene. Genet Med. 2001;3:91-101.

17. Hildmann T, Kong X, O'Brien J, et al. A contiguous 3-Mb sequenceready map in the S3-MX region on $21 \mathrm{q} 22.2$ based on highthroughput nonisotopic library screenings. Genome Res. 1999; 9:360-72.

18. Ascano M Jr., Mukherjee N, Bandaru P, et al. FMRP targets distinct mRNA sequence elements to regulate protein expression. Nature. 2012;492:382-6.

19. Brown V, Jin P, Ceman S, et al. Microarray identification of FMRPassociated brain mRNAs and altered mRNA translational profiles in fragile X syndrome. Cell. 2001;107:477-87.

20. Darnell JC, Van Driesche SJ, Zhang C, et al. FMRP stalls ribosomal translocation on mRNAs linked to synaptic function and autism. Cell. 2011;146:247-61.

21. Cvetkovska V, Hibbert AD, Emran F, Chen BE. Overexpression of Down syndrome cell adhesion molecule impairs precise synaptic targeting. Nat Neurosci. 2013;16:677-82.

22. Neufeld SQ, Hibbert AD, Chen BE. Opposing roles of PlexinA and PlexinB in axonal branch and varicosity formation. Molec Brain. 2011;4:15.

23. Kays I, Cvetkovska V, Chen BE. Structural and functional analysis of single neurons to correlate synaptic connectivity with grooming behavior. Nat Protocols. 2014;9:1-10.

24. Grossman TR, Gamliel A, Wessells RJ, et al. Over-expression of DSCAM and COL6A2 cooperatively generates congenital heart defects. PLoS Genet. 2011;7:e1002344.

25. Barlow G, Micales B, Chen X, Lyons GE, Korenberg J. Mammalian DSCAMs: roles in the development of the spinal cord, cortex, and cerebellum? Biochem Biophys Res Commun. 2002;293: 881-891.

26. Spruston N. Pyramidal neurons: dendritic structure and synaptic integration. Nat Rev Neurosci. 2008;9(3):206-21.

27. Somogyi P, Klausberger T. Defined types of cortical interneurone structure space and spike timing in the hippocampus. J Physiol. 2005;562:9-26.

28. Lo C, Kays I, Emran F, Lin T, Cvetkovska V, Chen BE. Quantification of Protein Levels in Single Living Cells. Submitted. 2015.

29. Suciu R, Aydin E, Chen BE. GeneDig: a web application for accessing genomic and bioinformatics knowledge. BMC Bioinformatics. 2015;16:16. 